\title{
CITOREDUCCIÓN GANGLIONAR LAPAROSCÓPICA EN RECIDIVA ÚNICA POR CÁNCERES GINECOLÓGICOS. EXPERIENCIA EN ARGENTINA
}

\section{LAPAROSCOPIC SURGERY FOR ISOLATED LYMPH NODES RECURRENCE IN GYNECOLOGY. EXPERIENCE IN ARGENTINA}

\author{
M. E. Giavedoni, ${ }^{1,2}$, J. M Saadi ${ }^{1}$, G. Saraniti ${ }^{1}$, M. Perrotta ${ }^{1}$
}

\section{Resumen:}

Objetivos: Describir los resultados perioperatorios y oncológicos de una serie de casos operados con laparoscopía por metástasis única ganglionar de cánceres ginecológicos en el Servicio de Ginecología del Hospital Italiano de Buenos Aires y realizar una revisión bibliográfica sobre el tema. Métodos: Se llevó a cabo una búsqueda de pacientes en los registros de cirugía del servicio de Ginecología del Hospital que hayan recibido tratamiento quirúrgico de citoreducción laparoscópica por recurrencia ganglionar aislada de un tumor primario ginecológico en el período 2009-2015. Se revisaron las historias clínicas y se recolectaron y analizaron datos referidos a características de las pacientes, del tumor, de la cirugía y sus complicaciones. Se realiza una búsqueda bibliográfica en Pubmed con el concepto "isolated nodal recurrence in gynecology" y posterior búsqueda en bola de nieve ${ }^{[1]}$. Resultados: Se presentan 9 pacientes. La mediana de edad fue de 66 años (RI 7). La localización del cáncer inicial fue ovario $(n=3)$, endometrio $(n=3)$, cuello $(n=2)$ y mama $(n=1)$. El sitio de localización de la recurrencia ganglionar única fue el área lumboaórtica $(n=8)$ y el área pelviana $(n=1)$. La mediana de intervalo libre de enfermedad a la recurrencia fue de 42.8 meses (RI 40.2). Se utilizó un abordaje retroperitoneal 0 transperitoneal según la localización de la recurrencia. La mediana de tiempo operatorio fue de 120 minutos (RI 36). No se realizó conversión a laparotomía en ninguna paciente. No se reportaron complicaciones intraoperatoria o postoperatorias, así como tampoco requerimiento de transfusión sanguínea. La mediana de estadía hospitalaria fue de 36 horas. Realizaron tratamiento adyuvante con quimioterapia 8/9 pacientes. Solo 1 paciente recurrió a nivel lumboaórtico y peritoneal luego de 8 meses y falleció de su enfermedad. La revisión de la literatura confirma la posibilidad de realizar la resección de la recurrencia ganglionar única por vía laparoscópica con baja tasa de complicaciones. Conclusión: la citorreducción ganglionar laparoscópica es una opción de tratamiento válida y segura en relación a los resultados perioperatorios y oncológicos en nuestra institución.

Palabras clave: laparoscopía; recurrencia ganglionar aislada; linfadenectomía; cáncer ginecológico.

\section{Abstract:}

Objectives: to describe the perioperative and oncological outcomes of a serie of cases treated with laparoscopic surgery for isolated nodal metastasis of gynecologic malignancies at the Gynecology Service of Hospital Italiano de Buenos Aires and conduct a literature review on the surgical treatment provided to patients with this condition. Methods: A search was conducted in the surgery records of the Gynecology Service of Hospital Italiano of patients who underwent laparoscopic cytoreductive surgery for isolated nodal recurrence of a gynecologic primary tumor in the period 2009-2015. The medical records were reviewed and data on the patients' characteristics, the tumor, the surgery and its complications were collected and analyzed. A literature search was conducted in Pubmed by the term "isolated nodal recurrence in gynecology" and a subsequent snowball search was performed. Results: As results, nine patients are presented. The median age was 66 years old (RI 7). The initial cancer location was: ovary $(n=3)$, endometrium $(n=3)$, cervix $(n=2)$ and breast $(n=1)$. The location site of the single nodal recurrence was the para-aortic area $(n=8)$ and the pelvic area $(n=1)$. The median of the disease-free interval to recurrence was 42.8 months (RI 40.2). Either the retroperitoneal or the transperitoneal approach was used based on the

1 Departamento de Ginecología, Sección de Ginecología Oncológica, Hospital Italiano de Buenos Aires, Argentina.

2 Email de contacto: maria.giavedoni@hospitalitaliano.org.ar

Recibido: 2017-06-02

Aceptado: 2017-10-10

DOI: 10.31053/1853.0605.v75.n2.17097 
location of the recurrence. The median surgery time was 120 minutes (RI 36). No conversion to laparotomy was performed in any patient. No intraoperative or postoperative complications were reported, nor any requirement for blood transfusion. The median hospital stay was 36 hours. Adjuvant treatment with chemotherapy was applied to 8/9 patients. Only one patient recurred in para-aortic lymph nodes and peritoneal carcinomatosis after eight months and died of her disease. The literature review confirms the possibility of laparoscopic resection of the single nodal recurrence with low complication rate. Conclusion: The laparoscopic lymph node cytoreductive surgery is a valid and safe treatment option considering the perioperative and oncological outcomes in our hospital.

Keywords: laparoscopy; isolated nodal recurrence; lymphadenectomy; gynecologic malignancy. 


\section{Introducción}

La recurrencia de un cáncer ginecológico en nódulos ganglionares, como único sitio de recaída, es poco frecuente, pero no excepcional. Generalmente este compromiso ganglionar se acompaña de compromiso de otros sitios del cuerpo, ya sea a nivel peritoneal u otras estructuras anatómicas. Sin embargo, ocasionalmente la recurrencia ganglionar aislada ocurre y sucede en aproximadamente el 2-4 $\%$ en los distintos cánceres ginecológicos ${ }^{[2][3] .}$

El manejo de estas recurrencias ganglionares aisladas no está claramente establecido y las posibilidades de tratamiento son variadas (quimioterapia, radioterapia y hormonoterapia), como así también los resultados terapéuticos. Aún no se ha podido aclarar cuál es el tratamiento más beneficioso en esta situación. La cirugía con fines citorreductivos puede ser considerada una buena alternativa en forma individual o acompañada, posteriormente, de alguna terapia adyuvante ${ }^{[4]}$.

A su vez, el abordaje laparoscópico aporta beneficios claros en relación a la laparotomía, que son ampliamente aceptados y conocidos, como los relacionados a un menor trauma, disminución de la hospitalización, menos pérdida de sangre y, con ello, menor requerimiento de transfusiones, menor requerimiento de analgesia postoperatoria y mejor calidad de vida. Una ventaja adicional y muy importante, radica en cuanto a la posibilidad de realizar tratamientos complementarios posteriores a la cirugía en forma más temprana. Esto es debido a la rápida recuperación por el menor trauma ocasionado ${ }^{[5][6]}$.

Dentro de la vía laparoscópica, existen dos formas de explorar el retroperitoneo: seccionando el peritoneo (transperitoneal) o ingresando al mismo sin incidir el peritoneo (extraperitoneal) ${ }^{[7]}$. El abordaje extraperitoneal tiene algunas ventajas con respecto al transperitoneal, en pacientes con cirugías previas abdominales, obesas, o cuando la localización de la recidiva está en el espacio paraaórtico ${ }^{[6]}$. Se requiere un experimentado cirujano con un elevado nivel de entrenamiento quirúrgico para poder realizar estas cirugías por vía laparoscópica, y es necesario que el mismo conozca las dos vías de abordaje (extra y transperitoneal), ya que de acuerdo al caso será de mayor utilidad una sobre la otra ${ }^{[8]}$.

Si bien existen comunicaciones de este tipo de procedimiento desde el año $1996{ }^{[7]}$ y aplicado a diferentes situaciones, es muy escasa los datos sobre la experiencia en América Latina. Por otro lado, el Servicio de ginecología del Hospital Italiano de Buenos Aires, Argentina, hace 6 años viene realizando este tipo de procedimientos laparoscópicos en pacientes con metástasis única de cáncer ginecológico.

Nos proponemos en este trabajo describir los resultados perioperatorios y oncológicos de una serie de casos operados con laparoscopía por metástasis única ganglionar de cánceres ginecológicos en el Servicio de Ginecología del Hospital Italiano de Buenos Aires, en el período 2010-2015, y realizar una revisión bibliográfica sobre tratamiento quirúrgico en pacientes con recidiva única en cáncer ginecológico.

\section{Materiales y Métodos}

Los datos fueron obtenidos de los registros de Cirugía del Servicio de Ginecología, Departamento de Ginecología Oncológica del Hospital Italiano de Buenos Aires, Argentina. Se llevó a cabo una búsqueda de pacientes que hayan recibido tratamiento quirúrgico de citoreducción laparoscópica por recurrencia ganglionar aislada de un tumor primario. Como práctica habitual del servicio, a todas las pacientes se les solicitó estudios de imágenes de complejidad: tomografia de emisión de positrones (PET-TC) o tomografía (TAC). Se incluyó pacientes con cualquier cáncer ginecológico primario: cáncer de ovario, endometrio, cuello y mama. Se excluyeron pacientes con cánceres concomitantes. Se revisaron las historias clínicas correspondientes a las pacientes seleccionadas y se recolectaron datos referidos a características de las pacientes, del tumor, de la cirugía y sus complicaciones.

Las variables correspondientes a las características de las pacientes al momento de la cirugía fueron la edad y el índice de masa corporal (BMI). Se analizaron las características del cáncer ginecológico inicial (localización, tipo histológico, tratamiento, extensión de cirugía y compromiso tumoral ganglionar) y las características de la recurrencia ganglionar aislada (sitio, método complementario de diagnóstico, tipo de cirugía, metástasis confirmada por histología, tamaño ganglionar). 
Como variables del tratamiento, se analizaron los resultados perioperatorios (duración de cirugía, complicaciones intraoperatorias y necesidad de transfusión, tiempo de internación, complicaciones postoperatorias) y tipo de tratamiento que recibió la paciente posteriormente.

Este estudio fue aprobado por el Comité de Evaluación de Protocolos del Hospital Italiano de Buenos Aires, Argentina (CEPI).

\section{Técnica quirúrgica}

A continuación, se describe la técnica quirúrgica realizada.

La cirugía fue realizaba bajo firma del consentimiento médico informado. Todos los procedimientos fueron realizados por el mismo equipo de médicos entrenados en laparoscopía y cirugías ginecológicas oncológicas.

La intervención se realiza bajo anestesia general e intubación orotraqueal. Se coloca a la paciente en posición supina y en posición de litotomía con las piernas semiflexionadas en abducción.

Los miembros superiores se colocan junto al cuerpo. La paciente se coloca en posición de Trendelemburg. Ambos ayudantes se colocan del lado izquierdo de la paciente.

Se inicia con el acceso a la cavidad abdominal con técnica de Opticview con trocar principal a nivel peri umbilical. Se visualiza la cavidad abdominal y el peritoneo.

En la práctica asistencial del servicio, optamos por el tipo de abordaje (trans o extraperitoneal) de acuerdo al sitio de recidiva.

Cuando realizamos el abordaje transperitoneal, colocamos las vías como se observa en la figura 1.

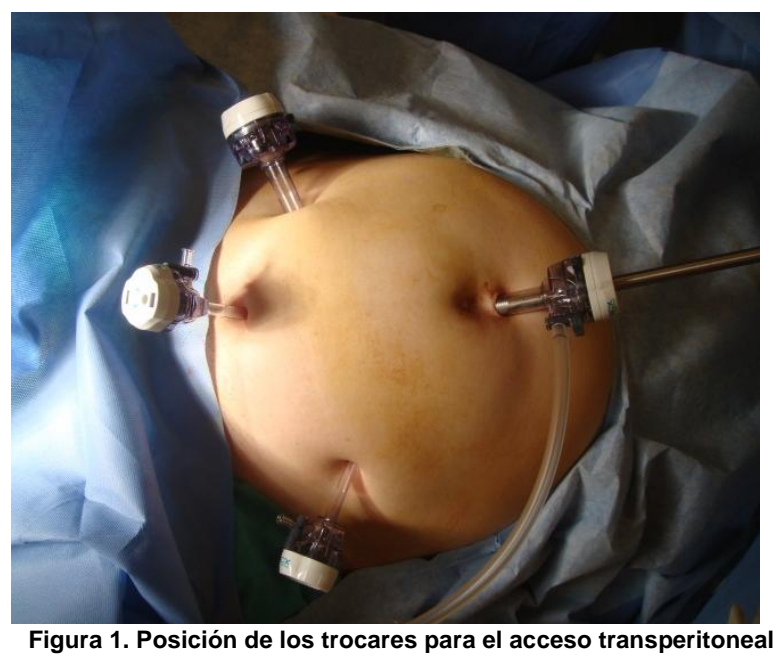

La vía principal de $10 \mathrm{~mm}$ supraumbilical a $2 \mathrm{~cm}$ a la izquierda. Las vías accesorias de $5 \mathrm{~mm}$ en fosa ilíaca izquierda y hipogastrio y vía de $10 \mathrm{~mm}$ en fosa ilíaca derecha. En cambio, cuando se realiza el acceso retroperitoneal las vías accesorias se colocan en la línea axilar media (Figura 2).

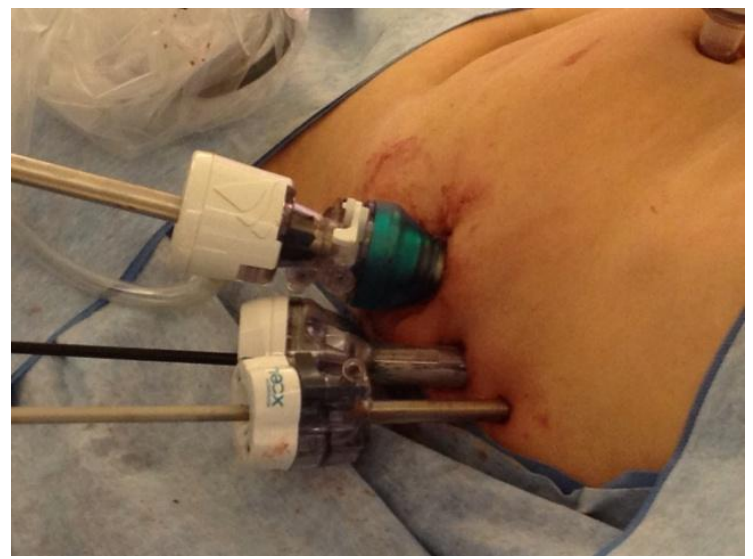

Figura 2. Posición de los trocares para el acceso retroperitoneal 
Primer paso para iniciar el procedimiento, por vía transperitoneal, es acceder al espacio retroperitoneal, incidiendo el peritoneo por encima de la arteria ilíaca primitiva derecha, dejando el uréter derecho adherido al peritoneo y expulsado hacia lateral.

Colocamos el endoretractor y exponemos los grandes vasos.

Una vez conformado el campo, procedemos a identificar el conglomerado ganglionar tumoral (figura 3).

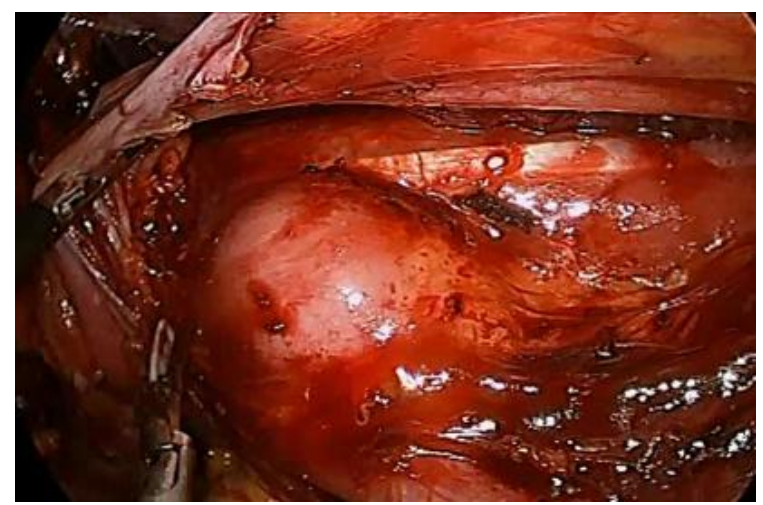

Figura 3. Identificación del conglomerado ganglionar, identificando estructuras vasculares adyacentes

Consideramos fundamental identificar el plano de clivaje entre el conglomerado y el vaso, mantener tracción suave y continua del mismo, en sentido contrario al vaso. Cuando es removido por completo lo retiramos en bolsa. El acceso transperitoneal es de elección cuando las lesiones se ubican paracavales o intercavoaórticas.

Para el abordaje extraperitoneal utilizamos la técnica descripta por Querleu ${ }^{[9]}$, para lo cual realizamos una incisión por encima de la espina iliaca izquierda, seccionamos los tejidos parietales por planos hasta llegar al peritoneo, el cual es mantenido indemne. Con maniobras digitales separamos el mismo sin dañarlo. Avanzamos en profundidad hasta palpar el resalto del músculo psoas y luego identificar el latido de la arteria ilíaca primitiva.

Luego colocamos la vía principal donde se coloca la óptica y, posteriormente bajo visión, colocamos 2 vías accesorias a la altura de la línea medio axilar con una separación entre ambas de $3 \mathrm{~cm}$ (figura 2). Comenzamos con la disección del espacio extraperitoneal. Una vez expuestas las estructuras vasculares, trabajamos sobre el conglomerado ganglionar. Se identifica la cara anterior y lateral de la Aorta.

Iniciamos primero la resección separando el conglomerado ganglionar del psoas. La tracción continua del conglomerado ganglionar nos permitirá mantener visible el plano de clivaje. Debemos resecar la masa ganglionar en forma completa.

Una vez extirpada la retiramos en bolsa. Cabe destacar que este abordaje es de preferencia cuando las recurrencias son paraaórticas. En los casos de confirmación histológica intraoperatoria de malignidad, no realizamos apertura del peritoneo, de esta forma evitar contaminación tumoral de la cavidad abdominal.

En todos los casos dejamos drenajes que son retirados al momento del alta hospitalaria.

\section{Análisis estadístico}

Se realiza un análisis descriptivo de las variables presentadas. Las variables continuas se expresaron como media y desvío estándar o mediana y rango intercuartilo según distribución. Las variables categóricas fueron presentadas como frecuencias absolutas, debido al bajo número de casos.

Para evaluar los resultados oncológicos, se estimaron el intervalo libre de enfermedad (definido como tiempo en meses transcurrido desde el diagnóstico de la enfermedad oncológica primaria hasta la cirugía de la recurrencia ganglionar aislada) y el intervalo libre de recurrencia (definido como tiempo desde la recurrencia ganglionar aislada hasta siguiente recaída tumoral o fecha de último control).

Se estimaron la tasa de recidiva como el número pacientes que recurrieron cada 1.000 personas-meses en seguimiento, con su respectivo intervalo de confianza del 95\%. El análisis estadístico se realizó con el programa STATA13. 


\section{Búsqueda bibliográfica}

Se realizó una búsqueda bibliográfica en Pubmed utilizando términos relacionados con el siguiente concepto: "isolated nodal recurrence in gynecology". A partir de los artículos encontrados, se utilizó una estrategia de búsqueda en bola de nieve, basándose en las referencias de los artículos referenciados.

\section{Resultados}

Se encontraron 9 pacientes a nuestro estudio, con historia de diferentes cánceres ginecológicos y recurrencia ganglionar retroperitoneal aislada. Los resultados de las características de las pacientes se encuentran resumidas en la Tabla 1.

Tabla 1. Características de las pacientes

\begin{tabular}{|c|c|}
\hline \multicolumn{2}{|l|}{ Variable } \\
\hline Edad (años) (mediana y rango intercuartilo) & $66(7)$ \\
\hline BMI (mediana y rango intercuartilo) & $29(10)$ \\
\hline \multicolumn{2}{|l|}{ Localización de cáncer inicial (frecuencia absoluta) } \\
\hline Ovario & $3 / 9$ \\
\hline Endometrio & $3 / 9$ \\
\hline Cuello & $2 / 9$ \\
\hline Mama & $1 / 9$ \\
\hline \multicolumn{2}{|l|}{ Tratamiento de cáncer inicial (frecuencia absoluta) } \\
\hline Cirugía & $7 / 9$ \\
\hline Radioterapia & $1 / 9$ \\
\hline Neoadyuvancia y radioterapia & $1 / 9$ \\
\hline \multicolumn{2}{|l|}{ Tipo histológico del tumor inicial } \\
\hline Carcinoma seroso & $4 / 8$ \\
\hline Carcinoma pavimentoso & $1 / 8$ \\
\hline Adenocarcinoma endometroide & $2 / 8$ \\
\hline Carcinona medular & $1 / 8$ \\
\hline \multicolumn{2}{|l|}{ Realización de linfadenectomía en cirugía inicial } \\
\hline $\mathrm{Si}$ & $2 / 6$ \\
\hline No & $4 / 6$ \\
\hline \multicolumn{2}{|l|}{ Tratamiento adyuvante luego de la cirugía inicial } \\
\hline Quimioterpia & $4 / 5$ \\
\hline Radioterpia & $1 / 5$ \\
\hline \multicolumn{2}{|l|}{ Sitio de la primer recurrencia oncológica } \\
\hline Ganglionar aislada & $5 / 9$ \\
\hline Otra localización & $4 / 9$ \\
\hline \multicolumn{2}{|l|}{ Método complementario diagnóstico de la recurrencia ganglionar única } \\
\hline Tomografía de emisión de positrones (PET-TC) & $8 / 9$ \\
\hline Tomografía (TAC) & $1 / 9$ \\
\hline Tamaño de la recurrencia ganglionar única $(\mathrm{mm})$ (mediana y rango intercuartilo) & $20(14)$ \\
\hline
\end{tabular}

Tabla 2. Resultados perioperatorios

\begin{tabular}{|lr|}
\hline Variable & \\
\hline Vía de acceso quirúrgico & $3 / 9$ \\
Transperitoneal & $6 / 9$ \\
\hline Retroperitoneal & $120(36)$ \\
\hline Duración de cirugía (minutos) (mediana y rango intercuartilo) & $36(24)$ \\
\hline Tiempo de estadía hospitalaria (horas) (mediana y rango intercuartilo) & $5(3.4)$ \\
\hline Número de ganglios obtenidos (media y desvío estandar)
\end{tabular}




\begin{tabular}{|lr|}
\hline Número de ganglios comprometidos (media y desvío estandar) & $3.4(2.4)$ \\
\hline Porcentaje de compromiso ganglionar (mediana y rango intercuartilo) & $83.33(40)$ \\
\hline Mayor tamaño de ganglio comprometido (mm) (media y desvío estandar) & $20.4(15.8)$ \\
\hline
\end{tabular}

Luego de la cirugía de citoreducción ganglionar, 8/9 pacientes realizaron quimioterapia.

Con respecto al intervalo libre de enfermedad, la mediana de tiempo a la recurrencia ganglionar única fue de 42.8 meses (rango intercuartilo 40.2).

La mediana de tiempo de seguimiento desde la enfermedad ganglionar aislada a la próxima recurrencia oncológica (Intervalo libre de recurrencia) fue de 15.3 meses (rango intercuartilo 9.3). La tasa de recaída fue de 6.4 pacientes cada 1000 personas- meses (IC 95\% 0.9-45,5).

Solo 1 paciente recurrió luego de la cirugía de citoreducción ganglionar aislada luego de 8 meses, a nivel retroperitoneal lumboaórtico y luego con carcinomatosis peritoneal y falleció de su enfermedad pasados 31 meses de su segunda recurrencia.

\section{Revisión de la bibliografía y discusión}

Se presenta la revisión de 12 artículos seleccionados por su relación directa con el tema seleccionado, publicados entre 2001-2014. Se desarrolla la comparación de los mismos en la tabla 3.

Tabla 3. Tabla comparativa

\begin{tabular}{|c|c|c|c|c|c|c|c|c|c|c|c|c|c|}
\hline Variable/Fuente & $\begin{array}{c}\text { Chou } \\
\text { (2001) } \\
{[10]}\end{array}$ & $\begin{array}{l}\text { Uzan } \\
\text { (2004 } \\
)[11]\end{array}$ & $\begin{array}{c}\text { Niibe } \\
\text { (2006) } \\
{[2]}\end{array}$ & $\begin{array}{c}\text { Blancha } \\
\text { rd } \\
(2007) \\
{[12]}\end{array}$ & $\begin{array}{c}\text { Santilla } \\
\mathbf{n} \\
(\mathbf{2 0 0 7 )} \\
{[13]}\end{array}$ & $\begin{array}{c}\text { San Juan } \\
\text { (2007) } \\
{[14]}\end{array}$ & $\begin{array}{c}\text { Legge } \\
(2008) \\
{[15]}\end{array}$ & $\begin{array}{c}\text { Gil- } \\
\text { Moreno } \\
\text { (2008) [5] }\end{array}$ & $\begin{array}{c}\text { Foutiou } \\
(2009) \\
{[16]}\end{array}$ & $\begin{array}{c}\text { Franco } \\
- \\
\text { Camps } \\
(2010) \\
{[6]} \\
\end{array}$ & $\begin{array}{c}\text { Hong } \\
\text { (2012) } \\
{[4]}\end{array}$ & $\begin{array}{c}\text { Ferrero } \\
\text { (2014) } \\
{[17]}\end{array}$ & $\begin{array}{l}\text { Nuestro } \\
\text { estudio }\end{array}$ \\
\hline Fecha & $\begin{array}{l}1990- \\
1996\end{array}$ & $\begin{array}{l}1987- \\
2000\end{array}$ & $\begin{array}{c}199 \\
4- \\
200 \\
3 \\
\end{array}$ & $\begin{array}{c}1986 \\
- \\
2001\end{array}$ & $\begin{array}{c}199 \\
7- \\
200 \\
5 \\
\end{array}$ & $\mathrm{FD}^{\mathrm{a}}$ & $\begin{array}{l}1995- \\
2006\end{array}$ & $\begin{array}{l}2002- \\
2007\end{array}$ & $\begin{array}{l}1995- \\
2007\end{array}$ & $\begin{array}{l}2002- \\
2009\end{array}$ & $\begin{array}{l}2003- \\
2010\end{array}$ & $\begin{array}{l}2000- \\
2012\end{array}$ & $\begin{array}{l}2010- \\
2015\end{array}$ \\
\hline $\mathbf{N}$ de pacientes & 26 & 12 & 84 & 27 & 25 & 7 & 32 & 8 & 21 & 15 & 6 & 73 & 9 \\
\hline \multirow[t]{2}{*}{ Sitio de cancer } & $*$ & * & $*$ & $*$ & $*$ & $*$ & $*$ & $*$ & $*$ & $*$ & * & $*$ & \\
\hline & Cervix & $\begin{array}{c}\text { Ovari } \\
\text { o }\end{array}$ & $\begin{array}{c}\text { Cerv } \\
\mathrm{x}\end{array}$ & $\begin{array}{c}\text { Ovar } \\
\text { io }\end{array}$ & $\begin{array}{c}\text { Ova } \\
\text { rio }\end{array}$ & Todos & $\begin{array}{c}\text { Ovari } \\
\text { o }\end{array}$ & Todos & Ovario & Todos & Todos & $\begin{array}{c}\text { Ovari } \\
\text { o }\end{array}$ & Todos \\
\hline Tratamiento inicial & $*$ & $*$ & $*$ & $*$ & $*$ & $*$ & $*$ & $*$ & * & $*$ & $*$ & * & $*$ \\
\hline Cirugia & 0 & 12 & 26 & 27 & 25 & 6 & 32 & 6 & 21 & 13 & 6 & 73 & 7 \\
\hline $\mathbf{Q T}^{\mathrm{b}}$ & 0 & 0 & 0 & 0 & 0 & 0 & 0 & 1 & 0 & 1 & 0 & 0 & $\mathbf{0}$ \\
\hline $\mathbf{R T}^{\mathrm{c}}$ & 26 & 0 & 42 & 0 & 0 & 0 & 0 & 1 & 0 & 1 & 0 & 0 & 2 \\
\hline QTRTe $^{\mathrm{d}}$ & 0 & 0 & 16 & 0 & 0 & 1 & 0 & 0 & 0 & 0 & 0 & 0 & $\mathbf{0}$ \\
\hline $\begin{array}{c}\text { Con/Sin linfadenectomia } \\
\text { lumboAortica }\end{array}$ & * & * & $*$ & $*$ & * & * & $*$ & $*$ & * & * & * & * & * \\
\hline Con & 0 & 3 & $\mathrm{FD}^{\mathrm{a}}$ & $\mathrm{FD}^{\mathrm{a}}$ & 15 & 3 & 10 & 6 & 9 & 13 & 5 & 42 & 2 \\
\hline Sin & 26 & 9 & $\mathrm{FD}^{\mathrm{a}}$ & $\mathrm{FD}^{\mathrm{a}}$ & $\mathrm{FD}^{\mathrm{a}}$ & 4 & 22 & 2 & 12 & 2 & 1 & 31 & 7 \\
\hline $\begin{array}{l}\text { Intervalo libre de enfermedad } \\
\text { (meses) (mediana, mím y máx) }\end{array}$ & $\begin{array}{l}24,1 \\
(5,7- \\
79,2) \\
\end{array}$ & $\begin{array}{c}21(6- \\
62)\end{array}$ & $\begin{array}{c}22 \\
(1- \\
103) \\
\end{array}$ & $\begin{array}{c}26 \\
(1- \\
159) \\
\end{array}$ & $\begin{array}{l}16 \\
(6- \\
40) \\
\end{array}$ & $\begin{array}{l}21 \\
(13- \\
46) \\
\end{array}$ & $\begin{array}{c}17.5 \\
(1- \\
134) \\
\end{array}$ & $\begin{array}{l}20(6- \\
96)\end{array}$ & $\begin{array}{c}21(8- \\
156)\end{array}$ & $\begin{array}{l}20(6- \\
96)\end{array}$ & $\begin{array}{l}32(12- \\
136)\end{array}$ & $\begin{array}{c}18(6- \\
192)\end{array}$ & $\begin{array}{l}42.8 \\
\left(\mathbf{R} I^{\mathrm{e}}\right. \\
40.2) \\
\end{array}$ \\
\hline $\begin{array}{l}\text { Sitio de RGA (recurrencia } \\
\text { ganglionar aislada) }\end{array}$ & $*$ & * & $*$ & $*$ & $*$ & $*$ & $*$ & $*$ & * & $*$ & * & * & $*$ \\
\hline LumboAortica & 26 & 7 & 84 & 15 & 16 & 6 & 23 & 8 & 12 & 15 & 6 & 37 & 8 \\
\hline Pelviana & 0 & 5 & 0 & 4 & 4 & 1 & 1 & 0 & 8 & 0 & 0 & 21 & 1 \\
\hline Otra & 0 & 2 & 0 & 14 & 6 & 0 & 8 & 0 & 5 & 0 & 0 & 3 & $\mathbf{0}$ \\
\hline Tratamiento inicial de RGA & $*$ & $*$ & * & $*$ & * & $*$ & $*$ & $*$ & $*$ & * & * & $*$ & $*$ \\
\hline Cirugia & 0 & 10 & 0 & 8 & 22 & 7 & 12 & 8 & 16 & 15 & 6 & 73 & 9 \\
\hline $\mathbf{Q T}^{\mathrm{b}}$ & 4 & 0 & 0 & 8 & 0 & 0 & 19 & 0 & $\mathrm{FD}^{\mathrm{a}}$ & 0 & 0 & 0 & $\mathbf{0}$ \\
\hline $\mathbf{R T}^{\mathrm{c}}$ & 1 & 0 & 52 & 2 & 0 & 0 & 0 & 0 & $\mathrm{FD}^{\mathrm{a}}$ & 0 & 0 & 0 & $\mathbf{0}$ \\
\hline QTRT $^{\mathrm{d}}$ & 14 & 0 & 32 & 2 & 0 & 0 & 0 & 0 & $\mathrm{FD}^{\mathrm{a}}$ & 0 & 0 & 0 & $\mathbf{0}$ \\
\hline Ninguna & 7 & 0 & 0 & 7 & 0 & 0 & 1 & 0 & $\mathrm{FD}^{\mathrm{a}}$ & 0 & 0 & 0 & 0 \\
\hline
\end{tabular}

\footnotetext{
${ }^{\mathrm{a}}$ Falta dato.

${ }^{\mathrm{b}}$ Quimioterapia

${ }^{\mathrm{c}}$ Radioterapia

${ }^{\mathrm{d}}$ Radioquimioterapia concurrente

${ }^{\mathrm{e}}$ Rango intercuantilo
} 
CITOREDUCCIÓN GANGLIONAR LAPAROSCÓPICA.

\begin{tabular}{|c|c|c|c|c|c|c|c|c|c|c|c|c|c|}
\hline Laparotomia & $N A^{f}$ & si & $*$ & $\mathrm{FD}^{\mathrm{a}}$ & si & 0 & $\mathrm{FD}^{\mathrm{a}}$ & no & si & no & no & $\mathrm{si}$ & 0 \\
\hline Laparoscopia & $\mathrm{NA}^{\mathrm{f}}$ & no & $*$ & $\mathrm{FD}^{\mathrm{a}}$ & no & 7 & $\mathrm{FD}^{\mathrm{a}}$ & si & no & si & si & no & 9 \\
\hline Retroperitoneal & $\mathrm{NA}^{\mathrm{f}}$ & $N A^{\mathrm{f}}$ & $N A^{\mathrm{f}}$ & $\mathrm{FD}^{\mathrm{a}}$ & $N A^{f}$ & 7 & $N A^{f}$ & $\mathrm{Si}$ & $N A^{\mathrm{f}}$ & si & $N A^{\mathrm{f}}$ & * & 6 \\
\hline Transperitoneal & $\mathrm{NA}^{\mathrm{f}}$ & $N A^{f}$ & $N A^{f}$ & $\mathrm{FD}^{\mathrm{a}}$ & $\mathrm{NA}^{\mathrm{f}}$ & 0 & $N A^{f}$ & $N A^{f}$ & $N A^{f}$ & $N A^{f}$ & si & $*$ & 3 \\
\hline $\begin{array}{c}\text { Conversion a laparotomia ( } \\
\text { número de casos) }\end{array}$ & $\mathrm{NA}^{\mathrm{f}}$ & $\mathrm{NA}^{\mathrm{j}}$ & $N A^{f}$ & $\mathrm{FD}^{\mathrm{a}}$ & 0 & 0 & $\mathrm{FD}^{\mathrm{a}}$ & 0 & $N A^{f}$ & 1 & 0 & $\mathrm{FD}^{\mathrm{a}}$ & 0 \\
\hline $\begin{array}{l}\text { Con/ Sin linfadenectomia } \\
\text { lumboAortica }\end{array}$ & $*$ & * & $*$ & $*$ & * & $*$ & $*$ & $*$ & $*$ & * & $*$ & * & $*$ \\
\hline Con & $N A^{f}$ & 10 & $\mathrm{NA}^{\mathrm{f}}$ & $\mathrm{FD}^{\mathrm{a}}$ & $\mathrm{FD}^{\mathrm{a}}$ & 5 & $\mathrm{FD}^{\mathrm{a}}$ & 8 & $\mathrm{FD}^{\mathrm{a}}$ & 15 & con & con & 3 \\
\hline Sin & $N A^{f}$ & 2 & $N A^{f}$ & $\mathrm{FD}^{\mathrm{a}}$ & $\mathrm{FD}^{\mathrm{a}}$ & 7 & $\mathrm{FD}^{\mathrm{a}}$ & 0 & $\mathrm{FD}^{\mathrm{a}}$ & 0 & $N A^{\mathrm{f}}$ & $N A^{f}$ & 7 \\
\hline $\begin{array}{l}\text { N de Ganglios obtenidos (media } \\
\text { [mínimo y máximo]) }\end{array}$ & $N A^{f}$ & $\mathrm{FD}^{\mathrm{a}}$ & $N A^{f}$ & $\mathrm{FD}^{\mathrm{a}}$ & $\begin{array}{c}1(1- \\
17)\end{array}$ & $\begin{array}{c}7.3(1- \\
15)\end{array}$ & $\mathrm{FD}^{\mathrm{a}}$ & $8(1-16)$ & $\mathrm{FD}^{\mathrm{a}}$ & $\begin{array}{c}7.7(1- \\
16) \\
\end{array}$ & $20(5-27)$ & $\begin{array}{c}16(2- \\
71)\end{array}$ & 5 \\
\hline $\begin{array}{c}\text { Porcentaje de ganglios positivos de } \\
\text { los extraídos (mediana) }\end{array}$ & $\mathrm{NA}^{\mathrm{j}}$ & $\mathrm{FD}^{\mathrm{K}}$ & $\mathrm{NA}^{\mathrm{j}}$ & $\mathrm{FD}^{\mathrm{k}}$ & $\begin{array}{c}100 \\
\%\end{array}$ & $100 \%$ & $\mathrm{FD}^{\mathrm{k}}$ & $21 \%$ & $\mathrm{FD}^{\mathrm{k}}$ & $20 \%$ & $47 \%$ & $\mathrm{FD}^{\mathrm{k}}$ & $\begin{array}{c}83.33 \\
\%\end{array}$ \\
\hline $\begin{array}{l}\text { Tiempo operatorio (min, mediana } \\
\text { [mínimo y máximo]) }\end{array}$ & $\mathrm{NA}^{\mathrm{j}}$ & $\mathrm{FD}^{\mathrm{k}}$ & $\mathrm{NA}^{\mathrm{j}}$ & $\mathrm{FD}^{\mathrm{k}}$ & $\mathrm{FD}^{\mathrm{k}}$ & $\begin{array}{c}207 \\
(120- \\
300) \\
\end{array}$ & $\mathrm{FD}^{\mathrm{k}}$ & $\begin{array}{l}157.5 \\
(120- \\
140)\end{array}$ & $\mathrm{FD}^{\mathrm{k}}$ & $\begin{array}{l}157.5 \\
(120- \\
240)\end{array}$ & $\begin{array}{c}337.5 \\
(200- \\
400)\end{array}$ & $\begin{array}{l}171 \\
(90- \\
450)\end{array}$ & 120 \\
\hline $\begin{array}{c}\text { Estadía hospitalaria (días, } \\
\text { mediana [mínimo y máximo]) }\end{array}$ & $\mathrm{NA}^{\mathrm{j}}$ & $\mathrm{FD}^{\mathrm{k}}$ & $\mathrm{NA}^{\mathrm{j}}$ & $\mathrm{FD}^{\mathrm{k}}$ & $\begin{array}{c}4(2- \\
10) \\
\end{array}$ & $\begin{array}{c}2.5(2- \\
3)\end{array}$ & $\mathrm{FD}^{\mathrm{k}}$ & $2(2-5)$ & $5(2-11)$ & $2(2-13)$ & $\begin{array}{c}8.5(5- \\
19)\end{array}$ & $\begin{array}{l}9(5- \\
10)\end{array}$ & 1.5 \\
\hline $\begin{array}{c}\mathbf{N} \text { de pacientes con complicaciones } \\
\text { intraoperatorias (número) }\end{array}$ & $\mathrm{NA}^{\mathrm{j}}$ & $\mathrm{FD}^{\mathrm{k}}$ & $\mathrm{NA}^{\mathrm{j}}$ & $\mathrm{FD}^{\mathrm{k}}$ & $\mathrm{FD}^{\mathrm{k}}$ & 0 & $\mathrm{FD}^{\mathrm{k}}$ & $\mathrm{FD}^{\mathrm{k}}$ & $\begin{array}{l}1 \text { (lesión } \\
\text { vascular) }\end{array}$ & 0 & $\begin{array}{l}1 \text { (lesión } \\
\text { vascular) }\end{array}$ & $\mathrm{FD}^{\mathrm{k}}$ & o \\
\hline $\begin{array}{c}\mathrm{N} \text { de pacientes con tranfusion } \\
\text { sanguínea ( } \mathrm{n} \text { absoluto) }\end{array}$ & $\mathrm{NA}^{\mathrm{j}}$ & $\mathrm{FD}^{\mathrm{k}}$ & $\mathrm{NA}^{\mathrm{j}}$ & $\mathrm{FD}^{\mathrm{k}}$ & $\mathrm{FD}^{\mathrm{k}}$ & 0 & $\mathrm{FD}^{\mathrm{k}}$ & 0 & $\mathrm{FD}^{\mathrm{k}}$ & 0 & $\mathrm{FD}^{\mathrm{k}}$ & $\mathrm{FD}^{\mathrm{k}}$ & 0 \\
\hline $\begin{array}{l}\mathrm{N} \text { de pacientes con complicaciones } \\
\text { postoperatorias (número) }\end{array}$ & $\mathrm{NA}^{\mathrm{j}}$ & $\mathrm{FD}^{\mathrm{k}}$ & $\mathrm{NA}^{\mathrm{j}}$ & $\mathrm{FD}^{\mathrm{k}}$ & $\mathrm{FD}^{\mathrm{k}}$ & 0 & $\mathrm{FD}^{\mathrm{k}}$ & $\mathrm{FD}^{\mathrm{k}}$ & $\mathrm{FD}^{\mathrm{k}}$ & $\begin{array}{c}1 \\
\text { (linfoce } \\
\text { le) }\end{array}$ & $\begin{array}{c}1 \\
\text { (linfede } \\
\text { ma) }\end{array}$ & $\mathrm{FD}^{\mathrm{k}}$ & 0 \\
\hline $\begin{array}{c}\text { Pacientes con tratamiento } \\
\text { adyuvante, (número absoluto) }\end{array}$ & * & * & * & * & * & * & * & * & * & * & * & * & * \\
\hline $\mathbf{Q T}^{\mathrm{g}}$ & $\mathrm{NA}^{\mathrm{j}}$ & 9 & $\mathrm{NA}^{\mathrm{j}}$ & 5 & 15 & 4 & $\mathrm{FD}^{\mathrm{k}}$ & 2 & 17 & 8 & 5 & 62 & 8 \\
\hline $\mathbf{R T}^{\mathrm{h}}$ & $\mathrm{NA}^{\mathrm{j}}$ & 1 & $\mathrm{NA}^{\mathrm{j}}$ & 1 & 4 & 3 & $\mathrm{FD}^{\mathrm{k}}$ & 4 & 1 & 8 & 0 & 0 & 1 \\
\hline QTRTe $^{\mathrm{i}}$ & $\mathrm{NA}^{\mathrm{j}}$ & 1 & $\mathrm{NA}^{1}$ & 0 & 4 & 0 & $\mathrm{FD}^{\mathrm{k}}$ & 1 & 3 & 0 & 1 & 0 & 0 \\
\hline Ninguna & $\mathrm{NA}^{1}$ & 1 & $\mathrm{NA}^{1}$ & 0 & 2 & 1 & $\mathrm{FD}^{\mathrm{k}}$ & 1 & 0 & 2 & 0 & 3 & 0 \\
\hline Sobrevida, media (meses) & $*$ & $*$ & $*$ & $*$ & $*$ & $*$ & $*$ & $*$ & * & * & $*$ & * & $*$ \\
\hline $\begin{array}{l}\text { Libre de recurrencia (mediana, } \\
\text { mínimo y máximo) }\end{array}$ & $\mathrm{NR}^{1}$ & $\begin{array}{l}44(8- \\
158)\end{array}$ & $\mathrm{NR}^{1}$ & 26 & 37 & $\begin{array}{l}12(6- \\
18)\end{array}$ & 37 & $\begin{array}{l}15.5(3- \\
49)\end{array}$ & 47 & $\begin{array}{l}16(0- \\
77)\end{array}$ & $\begin{array}{l}15(11- \\
68)\end{array}$ & 46 & $\begin{array}{c}15.3 \\
\left(\mathbf{R I}^{\mathrm{m}}\right. \\
\mathbf{9 . 3})\end{array}$ \\
\hline $\begin{array}{l}\text { Sobrevida global (mediana, } \\
\text { mínimo y máximo) }\end{array}$ & $\mathrm{NR}^{1}$ & $\begin{array}{l}114 \\
(43- \\
172)\end{array}$ & $\mathrm{NR}^{1}$ & $\begin{array}{c}68 \\
(12- \\
210)\end{array}$ & 61 & $\begin{array}{c}36 \\
(27- \\
59)\end{array}$ & 109 & $\begin{array}{c}28.5 \\
(23-99)\end{array}$ & 66 & $\mathrm{NR}^{1}$ & $\begin{array}{c}66(28- \\
151)\end{array}$ & $\mathrm{NR}^{1}$ & $\mathbf{N R}^{1}$ \\
\hline
\end{tabular}

Chou ${ }^{[10]}$ y Niibe ${ }^{[2]}$ reportaron su experiencia en pacientes con antecedente de cáncer de cuello. En el cáncer de ovario existen numerosas publicaciones que validan la citorreducción ganglionar en recurrencias aisladas: Uzan ${ }^{[11]}$, Santillan ${ }^{[13]}$, Fotiou ${ }^{[16]}$ y Ferrero ${ }^{[17]}$. Legge ${ }^{[15]}$ y Blanchard ${ }^{[12]}$, si bien reporta la experiencia de recurrencia aisladas en cáncer de ovario y afirman que este tipo de recurrencia tiene un patrón menos agresivo, no focalizan sus investigaciones en los resultados operatorios, sino que describen la heterogeneidad de tratamiento aplicados. Awtrey ${ }^{[18]}$ reporta su experiencia en el manejo de recurrencias en el cáncer de endometrio, pero un bajo porcentaje de sus pacientes podrían ser consideradas por su recurrencia ganglionar aislada. No puede generalizar los resultados a este grupo de nuestro estudio.

El resto de los artículos revisados $(n=4)$ presentaron características similares a nuestro estudio en cuanto a la diversidad de localización del tumor primario en las pacientes seleccionadas y similar tratamiento propuesto ${ }^{[4][6][5][14]}$.

Presentamos una serie de 9 pacientes con recurrencia ganglionar aislada intervenidas por citoreducción laparoscópica, sin complicaciones intraoperatorias o necesidad de conversión a laparotomía y con una mediana de sobrevida libre de recurrencia de 15.3 meses. Esta técnica quirúrgica se aplicó en pacientes con características diversas principalmente en cuanto al tratamiento previo, localización del cáncer inicial, localización de recurrencia.

La recurrencia ganglionar aislada, como único sitio de recurrencia en los distintos cánceres ginecológicos es poco frecuente y por lo tanto su modalidad terapéutica no está claramente establecida. En este sentido, a través de la revisión realizada en este estudio, encontramos escasas publicaciones

\footnotetext{
${ }^{\dagger}$ No aplica

${ }^{9}$ Quimioterapia

${ }^{\mathrm{h}}$ Radioterapia

Radioquimioterapia concurrente

jNo aplica

${ }^{\mathrm{k}}$ Falta dato

'No reporta

${ }^{\mathrm{m}}$ Rango intercuartilo
} 
con un bajo número de pacientes y con limitado valor estadístico para concluir sobre cuál es el mejor tratamiento en estos casos. Esto se debe a que la incidencia de compromiso ganglionar aislado como recurrencia de tumores ginecológicos es baja, siendo en cáncer de cuello del $2.1 \%{ }^{[2]}$, en cáncer de endometrio del $1.6 \%{ }^{[3]}$ y en el cáncer del ovario es del $1.1-4.2 \%{ }^{[19][12]}$.

En nuestro estudio, se consideró el tratamiento quirúrgico de la recurrencia ganglionar aislada como la opción más favorable de tratamiento primario, teniendo en cuenta la única localización tumoral, el tamaño del conglomerado tumoral y el entrenamiento quirúrgico. La cirugía también permitió descartar enfermedad en otro sitio o carcinomatosis peritoneal. Si bién en la mayoría de los estudios revisados se realiza citoreducción ganglionar, pocos son los que utilizan la vía laparoscópica ${ }^{[6] ~[5] ~[4] . ~}$

El abordaje laparoscópico puede ser considerado una alternativa para tratar quirúrgicamente estas recurrencias, por las ventajas que aporta la vía mínimamente invasiva en relación a los resultados perioperatorios, como así también acorta el período de tiempo entre la cirugía y el tratamiento adyuvante. Sin embargo, el abordaje laparoscópico para esta forma de recurrencia debe ser realizado por cirujanos con experiencia y un excelente entrenamiento. Por este tema, las publicaciones al respecto son limitadas a centros oncológicos que cuentan con este tipo de cirujanos ${ }^{[4]}{ }^{[8]}$.

En nuestra experiencia, se realizó el abordaje retroperitoneal o transperitoneal según la localización del conglomerado tumoral. En la revisión bibliográfica realizada, tanto la vía retroperitoneal ${ }^{[14][5][6]}$ como la vía transperitoneal ${ }^{[4]}$, fueron reportados por los diferentes autores. Según la experiencia de los casos reportados, la vía retroperitoneal resultó una excelente alternativa, al evitar el contacto tumoral con la cavidad abdominal, reducir el riesgo de lesiones intestinales, brindar una mejor exposición del área paraaórtica y evitar adherencias intraabdominales, crucial en las pacientes que realizarán radioterapia posterior.

El tiempo quirúrgico en nuestra serie presentó una mediana de 120 minutos, siendo menor a lo reportado en la bibliografía para abordaje laparoscópico ${ }^{[6][5][4]}$. Esto puede ser justificado por el manejo habitual de la vía laparoscópica en nuestra institución y debido a que todas las pacientes fueron operadas por el mismo cirujano entrenado. Por otro lado, la mediana de estadía hospitalaria fue menor

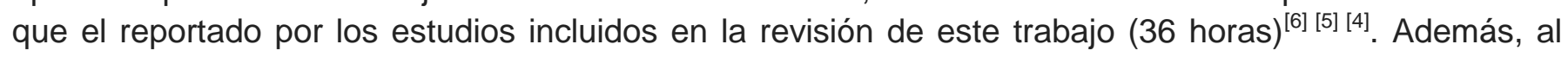
igual que en este estudio, pocas series han reportado la ausencia de complicaciones mayores en el procedimiento de citoreducción ganglionar por laparoscopía ${ }^{[6][5]}$.

Con respecto a las indicaciones de la cirugía, a diferencia de otros estudios, este estudio incluye los resultados de una paciente con recurrencia ganglionar pelviana aislada, lo cual aporta conocimiento en esta localización. En nuestro estudio, la obesidad no representó una contraindicación, a pesar de considerarse una cirugía técnicamente mas laboriosa, como lo refieren algunos autores ${ }^{\text {[20] }}$.

Con respecto a los resultados oncológicos, el intervalo libre de enfermedad (mediana= 42 meses) en esta serie fue mayor que publicaciones revisadas, excepto los trabajos que incluyeron solamente pacientes con cáncer de ovario ${ }^{[13][15][17]}$. Esto puede deberse, a que consideramos el tiempo a la recurrencia ganglionar aislada e incluimos pacientes con localizaciones primarias diversas. Por otro lado, el tiempo libre de recurrencia (mediana=15.3 meses) fue similar que lo reportado en algunos trabajos, que poseen los mismos criterios de selección de pacientes y de tipo de tratamiento realizado

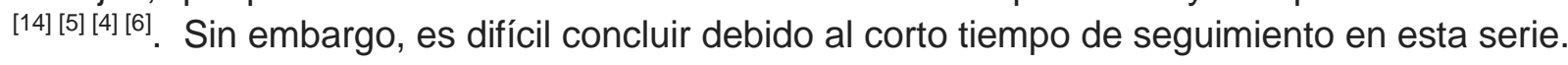

Como limitaciones al estudio, podemos mencionar el bajo número de pacientes y el corto tiempo de seguimiento de las mismas, lo que podría influir en la tasa de recidiva estimada. A su vez, este estudio se desarrolla en un hospital de alta complejidad, con médicos entrenados en laparoscopía y en oncología, lo que dificultaría la generalización de los resultados.

\section{Conclusión}

El uso de la laparoscopía en el manejo de las masas ganglionares es de mucha utilidad, porque además de permitir realizar la citorreducción completa de las mismas, otorga beneficios adicionales relacionados a la técnica laparoscópica y permite comenzar en menor tiempo el tratamiento adyuvante. Cabe remarcar la complejidad de este procedimiento ya que, las complicaciones fundamentalmente vasculares, pueden ser severas y requieren de mucha experiencia para resolverlas. Según la 
experiencia hasta el momento en nuestra institución, el mismo resultó ser una opción de tratamiento válida y segura para estas pacientes.

Si bien hacen falta más estudios con mayor número de pacientes y estudios comparativos con distintas opciones de tratamiento para concluir sobre el abordaje terapéutico más adecuado, los resultados de esta investigación pretenden contribuir al estudio y conocimiento sobre el tratamiento en la recurrencia ganglionar aislada de cánceres ginecológicos.

\section{Bibliografía}

[1] J. Higgins and S. Green, "Manual Cochrane de revisiones sistemáticas de intervenciones," Cochrane Handb. Syst. Rev. Interv., vol. Version 5., no. March, pp. 1-639, 2011.

[2] Y. Niibe, T. Kazumoto, T. Toita, H. Yamazaki, K. Higuchi, N. Ii, K. Suzuki, T. Uno, S. Tokumaru, M. Takayama, K. Sekiguchi, Y. Matsumoto, K. Michimoto, M. Oguchi, and K. Hayakawa, "Frequency and characteristics of isolated para-aortic lymph node recurrence in patients with uterine cervical carcinoma in Japan: A multi-institutional study," Gynecol. Oncol., vol. 103, no. 2, pp. 435-438, 2006.

[3] N. R. Abu-Rustum, J. D. Gomez, K. M. Alektiar, R. A. Soslow, M. L. Hensley, M. M. Leitao, G. J. Gardner, Y. Sonoda, D. S. Chi, and R. R. Barakat, "The incidence of isolated paraaortic nodal metastasis in surgically staged endometrial cancer patients with negative pelvic lymph nodes," Gynecol. Oncol., vol. 115, no. 2, pp. 236-238, 2009.

[4] J. H. Hong, J. S. Choi, J. H. Lee, J. W. Bae, J. M. Eom, J. T. Kim, and S. Oh, "Laparoscopic Lymphadenectomy for Isolated Lymph Node Recurrence in Gynecologic Malignancies," J. Minim. Invasive Gynecol., vol. 19, no. 2, pp. 188195, 2012.

[5] A. Gil-Moreno, S. Franco-Camps, B. Diaz-Feijoo, A. Perez-Benavente, J. Martinez-Palones, J. Del Campo, P. M., V. R., C. J., and X. J., "Usefulness of extraperitoneal laparoscopic paraaortic lymphadenectomy for lymph node recurrence in gynecologic malignancy," Acta Obstet. Gynecol., vol. 87, no. 7, pp. 723-730, 2008.

[6] S. Franco-Camps, S. Cabrera, A. Perez-Benavente, B. Díaz-Feijoo, M. Bradbury, J. Xercavins, and A. Gil-Moreno, "Extraperitoneal Laparoscopic Approach for Diagnosis and Treatment of Aortic Lymph Node Recurrence in Gynecologic Malignancy," J. Minim. Invasive Gynecol., vol. 17, no. 5, pp. 570-575, 2010.

[7] S. A. Vasilev and K. F. McGonigle, "Extraperitoneal laparoscopic paraaortic lymph node dissection: development of a technique.," J. Laparoendosc. Surg., vol. 5, no. 2, pp. 85-90, 1995.

[8] P. T. Ramirez, "Laparoscopic Surgery for Isolated Nodal Recurrence: Appropriate for All Patients or Only a Select Few?," J. Minim. Invasive Gynecol., vol. 19, no. 2, pp. 146-147, 2012.

[9] D. Querleu, G. Ferron, A. Rafii, E. Bouissou, M. Delates, E. Mery, and L. Gladieff, "Pelvic lymph node dissection via a lateral extraperitoneal approach: description of a technique.," Gynecol oncol, vol. 109, no. 1, pp. 81-85, 2008.

[10] H. H. Chou, C. C. Wang, C. H. Lai, J. H. Hong, K. K. Ng, T. C. Chang, C. J. Tseng, C. S. Tsai, and J. T. Chang, "Isolated paraaortic lymph node recurrence after definitive irradiation for cervical carcinoma," Int. J. Radiat. Oncol. Biol. Phys., vol. 51, no. 2, pp. 442-448, 2001.

[11] C. Uzan, P. Morice, A. Rey, P. Pautier, S. Camatte, C. Lhommé, C. Haie-meder, P. Duvillard, and D. Castaigne, "Outcomes After Combined Therapy Including Surgical Resection in Patients with Epithelial Ovarian Cancer Recurrence ( s ) Exclusively in Lymph Nodes," Ann. Surg. Oncol., vol. 11, no. 7, pp. 658-664, 2004.

[12] P. Blanchard, A. Plantade, C. Pagès, P. Afchain, C. Louvet, C. Tournigand, and A. de Gramont, "Isolated lymph node relapse of epithelial ovarian carcinoma: Outcomes and prognostic factors," Gynecol. Oncol., vol. 104, no. 1, pp. 41-45, 2007.

[13] A. Santillan, A. K. Karam, A. J. Li, R. Giuntoli, G. J. Gardner, I. Cass, B. Y. Karlan, and R. E. Bristow, "Secondary cytoreductive surgery for isolated nodal recurrence in patients with epithelial ovarian cancer $\vec{\sim}$," Gynecol. Oncol., vol. 104, no. 2007, pp. 686-690, 2007.

[14] A. Sanjúan, A. Illa, A. Torné, S. Martinez Román, M. Jurado, J. A. Lejarcegui, and J. Pahisa, "Extraperitoneal laparoscopic para-aortic lymphadenectomy as a diagnostic procedure for lymph node recurrence of gynaecological cancers," Acta Obstet. Gynecol., vol. 86, no. January, pp. 491-495, 2007.

[15] F. Legge, M. Petrillo, V. Adamo, S. Pisconti, G. Scambia, and G. Ferrandina, "Epithelial ovarian cancer relapsing as isolated lymph node disease: natural history and clinical outcome., "BMC Cancer, vol. 12, no. 8, p. 367, 2008.

[16] S. Fotiou, T. Aliki, Z. Petros, S. loanna, V. Konstantinos, M. Vasiliki, and C. George, "Secondary cytoreductive surgery in patients presenting with isolated nodal recurrence of epithelial ovarian cancer," Gynecol. Oncol., vol. 114, no. 2, pp. 178-182, 2009.

[17] a Ferrero, A. Ditto, G. Giorda, A. Gadducci, S. Greggi, A. Daniele, L. Fuso, E. Panuccio, C. Scaffa, F. Raspagliesi, $P$. Sismondi, and N. Biglia, "Secondary cytoreductive surgery for isolated lymph node recurrence of epithelial ovarian cancer: a multicenter study.," Eur. J. Surg. Oncol., vol. 40, no. 7, pp. 891-8, 2014.

[18] C. S. Awtrey, M. G. Cadungog, M. M. Leitao, K. M. Alektiar, C. Aghajanian, A. J. Hummer, R. R. Barakat, and D. S. Chi, "Surgical resection of recurrent endometrial carcinoma," Gynecol. Oncol., vol. 102, no. 3, pp. 480-488, 2006.

[19] P. Morice, F. Joulie, S. Camatte, D. Atallah, R. Rouzier, P. Pautier, C. Pomel, C. Lhommé, P. Duvillard, and D. Castaigne, "Lymph node involvement in epithelial ovarian cancer: Analysis of 276 pelvic and paraaortic lymphadenectomies and surgical implications," J. Am. Coll. Surg., vol. 197, no. 2, pp. 198-205, 2003.

[20] E. Leblanc, F. Narducci, M. Frumovitz, A. Lesoin, B. Castelain, M. C. Baranzelli, S. Taieb, C. Fournier, and D. Querleu, "Therapeutic value of pretherapeutic extraperitoneal laparoscopic staging of locally advanced cervical carcinoma," Gynecol. Oncol., vol. 105, no. 2007, pp. 304-311, 2007. 